\title{
EIN FAVELA-MUSICAL ÜBERSETZEN: VINICIUS DE MORAES' ORFEU DA CONCEIÇÃO AUF DEUTSCH
}

\author{
Martina Kopf ${ }^{1}$ \\ 1Johannes-Gutenberg-Universität Mainz, Mainz, Alemanha
}

Resumo: Vinicius de Moraes’ Musiktheaterstück Orfeu da Conceição. Tragédia carioca em três atos (1954) kann sowohl als Klassiker brasilianischer Literatur als auch als Weltliteratur betrachtet werden. Vinicius verknüpft den griechischen Stoff mit afrobrasilianischer Kultur, Musikstücken, die später zu Bossa Nova-Klassiker wurden und inszeniert einen schwarzen Orpheus auf einem Favelahügel in Rio de Janeiro. Das Stück wurde zwar mehrfach verfilmt, aber kaum übersetzt. Der Beitrag möchte ein paar Herausforderungen für die Übersetzung ins Deutsche skizzieren. Vor allem für die Vermittlung afrobrasilianischer Kultur müssen Strategien entwickelt werden. Erstens soll es um das semantische Feld "Favela" und den Favela-Slang gehen, zweitens um die Übersetzung der Musikstücke und das semantische Feld "Samba". Es zeigt sich als sinnvoll, Begriffe, die auf einen spezifisch brasilianischen soziokulturellen Kontext verweisen, im Original zu belassen, so zum Beispiel das Diminutiv neguinho/neguinha oder Samba-Musikinstrumente. Eine weitere Möglichkeit stellt die Anpassung eines Begriffs (morro) ins Deutsche (Favelahügel) dar, um die soziokulturelle Bedeutung der Ausgangssprache in die Zielsprache zu transportieren. Diese Strategien tragen dazu bei, den spezifisch brasilianischen bzw. afrobrasilianischen Charakter des Stücks auch in der Übersetzung zu betonen, wobei vor allem dem Paratext eine kulturvermittelnde Funktion zukommt.

Palavras-chave: Vinicius de Moraes; Orfeu da Conceição; Übersetzung; Afrobrasilianische Kultur 


\title{
TRANSLATING A FAVELA MUSICAL: VINICIUS DE MORAES' ORFEU DA CONCEIÇÃO IN GERMAN
}

\begin{abstract}
Vinicius de Moraes' musical Orfeu da Conceição. Tragédia carioca em três atos (1954) can be considered both a classic of Brazilian literature and world literature. Vinicius links the Greek myth with AfroBrazilian culture, musical pieces that became bossa nova classics, and stages a black Orpheus on a favela hill in Rio de Janeiro. The play has been picturized several times but has been hardly translated. This article would like to outline a few challenges for a translation into German. First and foremost, strategies need to be developed for conveying Afro-Brazilian culture. Firstly, the semantic field "favela" and the favela slang will be examined, secondly, the translation of the musical pieces and the semantic field "samba". It turns out to be useful to leave terms in the original which refer to a specific Brazilian socio-cultural context, for example the diminutive neguinho/neguinha or samba instruments. Another possibility is the adaptation of a term (morro) in German (favela hill) in order to transport the socio-cultural meaning from the source language to the target language. These strategies help to emphasize a specific Brazilian or AfroBrazilian character of the piece in translation as well. In this context, the paratext has a culture-mediating function.
\end{abstract}

Keywords: Vinicius de Moraes; Orfeu da Conceição; Übersetzung; Afrobrasilianische Kultur

\section{Ein Favela-Musical übersetzen: Vinicius de Moraes' Orfeu da Conceição auf Deutsch}

Im Sommersemester 2016 veranstaltete ich ein Seminar zum Thema "Orpheus weltweit. Orpheus-Rezeptionen in Afrika, Asien, Lateinamerika und den USA". Mir ging es darum, einen komparatistischen Blick auf außereuropäische Rezeptionen des antiken Mythos zu werfen. Durch Marcel Camus' preisgekrönten Film Orfeu Negro (1959) ${ }^{1}$ wurde ich auf die Vorlage aufmerksam, nämlich Vinicius de Moraes' Musiktheaterstück Orfeu da

${ }^{1}$ Die Verfilmung wurde 1960 in Cannes mit der Goldenen Palme ausgezeichnet. 
Conceição. Tragédia carioca em três atos (1954). Musiktheater mag heute etwas veraltet klingen, es handelt sich eigentlich um eine Art Musical, also ein Theaterstück mit Bossa Nova - Klassikern, das afrobrasilianische Kultur mit dem griechischen Stoff verknüpft und einen schwarzen Orpheus ${ }^{2}$ auf einem Favela-Hügel in Rio de Janeiro inszeniert. 1956 kam es in Rio de Janeiro zur Uraufführung und zum ersten Mal betraten schwarze Schauspieler*innen die Bühne des Theatro Municipal. Die Musikstücke schrieb Vinicius in Zusammenarbeit mit Antônio Carlos Jobim, Oscar Niemeyer wirkte als Bühnenbildner und Carlos Scliar als Plakatzeichner. Leider liegt bisher keine deutsche Übersetzung ${ }^{3}$ dieses brasilianischen Klassikers vor und da die Studierenden meines Seminars keine Portugiesisch-Kenntnisse hatten, entschloss ich mich kurzerhand, das Stück zu übersetzen. Die Arbeit an dieser 2016 entstandenen Rohfassung habe ich wieder aufgenommen.

Das Stück ist für ein deutsches Publikum aus verschiedenen Gründen interessant: Erstens handelt es sich um einen Klassiker brasilianischer Literatur, der durch seine produktive Rezeption des Orpheus-Stoffes, seine Funktion als Filmvorlage und aufgrund der transkulturellen Thematik der Weltliteratur zugeordnet werden kann. Zweitens gewinnt das Stück, das einen frühen Versuch darstellt, die schwarze Bevölkerung und afrobrasilianische Kultur in die brasilianische Gesellschaft zu integrieren, vor dem Hintergrund von Migration und Integration, Diskriminierung und

2 Vinicius scheint damit dem Trend eines schwarzen Orpheus, also einer Verbindung zwischen Orpheus-Mythos und afrikanischer Kultur, zu folgen: 1954 erschien die Lyrikanthologie Schwarzer Orpheus. Moderne Dichtung afrikanischer Völker beider Hemisphären von Janheinz Jahn. Der Titel bezieht sich auf Sartres berühmtes Vorwort von Léopold Sédar Senghors Anthologie de la nouvelle poésie nègre et malgache de langue française (1948). Vgl. dazu Porra, Véronique: "Sur quelques Orphée noirs. Reproduction, adaptation et hybridation du mythe d'Orphée en contexte post (-) colonial". Revue de Littérature Comparée 4 (2012): 441-455.

${ }^{3}$ Ins Italienische wurde das Stück sehr schnell übersetzt: Moraes, Vinicius de. Orfeo Negro. Tradução de Pasquale Aniel Jannini. Milano: Nuova Accademia Editrice, 1961.

Cad. Trad., Florianópolis, v. 41, no 3 p. 179-200, set-dez, 2021. 
Ungleichheit sowie vor allem der Black Lives Matter -Bewegung in einem weltweiten Kontext an Aktualität. Drittens ist Orfeu da Conceição nicht nur als literarisches und kulturhistorisches Dokument interessant - das Stück und seine Verfilmung verhalfen beispielsweise dem Bossa Nova zu einem internationalen Erfolg -, sondern auch als Vorlage für zukünftige internationale Inszenierungen mit neuen Gestaltungsideen. Gleich zu Beginn seines Stücks weist Vinicius nämlich darauf hin, dass Orfeu da Conceição stets aktualisiert werden solle (Moraes, Orfeu, 54).

Dass Aktualisierungen bzw. Adaptionen Herausforderungen darstellen und nicht immer den Vorstellungen der Autor*innen entsprechen, musste Vinicius selbst erleben. Wie viele andere, ${ }^{4}$ zeigte er sich über Camus' Orfeu Negro empört: "Essa história de puxar pelo exótico não pegou nada bem" (Castello 195). Herausforderungen zeigen sich nicht nur bei der Inszenierung, sondern auch bei der Übersetzung: Mit welchen sprachlichen und kulturhistorischen Herausforderungen muss sich eine Übersetzung ins Deutsche ca. 50 Jahre später auseinandersetzen? Vinicius' Texte wurden kaum ins Deutsche übersetzt, ${ }^{5}$ das mag vielleicht an der kaum übersetzbaren formalen Gestaltung seiner Verse liegen. Um Reim oder Rhythmus soll es hier an erster Stelle aber nicht gehen, vielmehr soll der Blick auf die Übersetzbarkeit einiger Begriffe aus dem afrobrasilianischen Kontext geworfen werden.

Nach einer kurzen Einführung zu Orfeu da Conceição möchte ich ein paar dieser Herausforderungen skizzieren: Erstens soll es um das semantische Feld "Favela" und den Favela-Slang gehen,

\footnotetext{
${ }^{4}$ So zum Beispiel Carlos Diegues, der 1999 mit Orfeu eine weitere Verfilmung wagte und sich kritisch gegenüber Camus' Verfilmung äußerte: “ [...] no Orfeu negro todo mundo pula e dança-, um lugar no mundo onde as pessoas são lindas, que só fazem dançar e cantar, e que o único grilo e a existência da morte" (Diegues 15).

${ }^{5}$ Kay-Michael Schreiner scheint der einzige zu sein, der eine Auswahl an Lyrik und Songs ins Deutsche übertragen hat. Vgl. Vinicius de Moraes. Saravá. Gedichte und Lieder, selecionado e traduzido por Kay-Michael Schreiner, Frankfurt: Vervuert, 1982.
} 
zweitens um die Übersetzung der Musikstücke und das semantische Feld "Samba".

\section{Orfeu da Conceição. Tragédia carioca em três atos (1954)}

Vinicius de Moraes (1913-1980), Diplomat, Dichter und Musiker, wurde bei einem alle Schichten der brasilianischen Gesellschaft umfassenden Publikum vor allem durch seine Verbindung zwischen Literatur und Musik beliebt (Schreiner 7). Er schrieb Sonette wie auch die Textvorlagen für Bossa Nova Klassiker und arbeitete mit prominenten Musikern wie Tom Jobim, Toquinho oder Baden Powell zusammen. 1942 begann er mit der Arbeit an Orfeu da Conceição: "Ich bin immer Musiker gewesen, aber ich fühlte mich vor allem als Dichter. Daher mein Interesse an dem Mythos des Orfeus [sic] - des Dichter-Musikers, den ich im idealen Sinn für den großen schöpferischen Geist hielt" (Moraes, Gespräch, 113). Die Liaison von Literatur und Musik setzte er dann auch stilistisch um, nämlich in Form eines Theaterstücks in Versen mit Musik- und Gesangseinlagen.

Die Idee zu einem schwarzen Orpheus in einer Favela kam Vinicius, während er im Haus seines Schwagers, des Architekten Carlos Leão, in Niterói den Mythos von Orpheus las und seine Lektüre von einer batucada begleitet wurde, die vom ganz in der Nähe gelegenen Morro do Cavalão hinüber hallte:

\footnotetext{
De súbito, as duas ideias ligaram-se no meu pensamento, e a vida do morro, com seus heróis negros tocando violão, e suas paixões, e suas escolas de samba que descem à grande cidade durante o Carnaval, e suas tragédias passionais, me pareceu tão semelhante à vida do divino músico negro, e à eterna lenda da sua paixão e morte, que comecei a sonhar um Orfeu negro. (Moraes, Radar, 48)
} 
Es war außerdem sein US-amerikanischer Schriftstellerkollege Waldo Frank, der ihn dazu brachte, die Welt "der Schwarzen" in Brasilien wahrzunehmen. ${ }^{6}$ Mit ihm reiste er durch Brasilien, entdeckte nicht nur "favelas, macumbas, clubes e festejos negros no Rio", sondern "candomblés, capoeiras e festejos negros da Bahia" und fühlte sich schließlich "impregnado do espírito da raça” (Moraes, Radar, 47). Diese Festivitäten der schwarzen Kultur vergleicht er mit der Kultur und den Traditionen der alten Griechen. ${ }^{7}$ Den "Schwarzen" bezeichnet Vinicius als "grego em ganga" (Moraes, Radar, 47) und schreibt ihm eine eigene Kultur zu:

"O negro possui uma cultura própria e um temperamento sui generis e embora integrado no complexo racial brasileiro, sempre manifestou a necessidade de seguir a trilha de sua própria cultura, prestando assim uma contribuição verdadeiramente pessoal à cultura brasileira em geral; aquela liberta dos preconceitos de cor, credo e classe" (Moraes, Radar, 49).

Diese Gedanken finden sich in einem, in den brasilianischen Ausgaben von 1995, 2013 und 2016, dem Stück vorangestellten Text

${ }^{6}$ Es war vor allem die Begegnung mit Waldo Frank, die Vinicius' politische Einstellung veränderte: "Bis zu seiner Begegnung mit dem Marxisten Waldo Frank hegte Moraes, wie auch andere Intellektuelle im Staatsdienst der Vargas-Zeit, diffuse Sympathien für die faschistische Allianz zwischen Hitler und Mussolini. Auch vor diesem ideologischen Hintergrund war die Welt der Schwarzen für den weißen Brasilianer eine gänzlich fremde. Den urbanen Raum der Favelas oben auf den Hügeln erkennt Moraes nicht als Folge der geographischen Exklusion von Armen und Schwarzen, sondern lediglich im Unterschied zu seiner Welt, der des Asphalts in den südlichen Stadtvierteln nahe am Strand" (Armbruster, "Neue Heimat, altes Elend - Das Eigene und das Fremde in brasilianischen und europäischen Diskursen über die Favela”, 379).

${ }^{7}$ Als er mit Waldo Frank die Favela da Praia do Pinto besuchte, verglich dieser die Bewohner*innen mit Griech*innen: "Eles parecem gregos diz. 'Gregos antes da cultura grega'" (Castello 182). 
mit dem Titel Radar da Batucada, der in der deutschen Übersetzung ebenfalls berücksichtigt wird. ${ }^{8}$ Dieser Paratext vermittelt nicht nur wichtige Informationen zur Entstehungsgeschichte, sondern verdeutlicht Vinicius' Anliegen einer Integration schwarzer Kultur. Paradoxerweise scheint das Theaterstück diesen Thesen allerdings an manchen Stellen zu widersprechen. ${ }^{9}$

Wie der Titel bereits andeutet, handelt es sich bei Orfeu da Conceição um eine Tragödie in drei Akten: Der erste Akt porträtiert den Musiker Orfeu und seine Eltern Clio und Apolo in ihrer Hütte auf einem Favela-Hügel in Rio de Janeiro. Neben seiner Geliebten Eurídice tritt auch eine ehemalige Geliebte Orfeus auf, "Mira de tal, mulher do morro". Der erste Akt endet mit dem dramatischen Mord Aristeus an Eurídice. Ihr Tod kündigt sich in personifizierter Form durch die "Dama Negra" an: "Hoje alguém me chamou que vai comigo/ Para o fundo da noite vai comigo/ Alguém que me chamou" (Moraes, Orfeu, 76). Orfeus Versuche, sie zum Rückzug zu bewegen, scheitern: Eurídice stirbt. Im zweiten Akt, der erst sechs Jahre später entstand, steigt Orfeu in die Unterwelt hinab, die durch den "Carnaval carioca" repräsentiert wird. Hier feiern die Mitglieder des Vereins "Os Maiorais do Inferno" einen "baile de terça-feira gorda”, wo Plutão als "presidente dos Maiorais do Inferno" im Mittelpunkt thront, wie es in der Regieanweisung heißt: "Num trono diabólico, ao fundo, sentam-se Plutão e Prosérpina, com uma corte de mulheres à volta. Esse casal mefistofélico deve se caracterizar pelo tamanho e gordura, gente gigantesca, risonha, desperdiçada, a aproximar comparsas solitários, a gritar,

${ }^{8}$ Es handelt sich um eine Montage von zwei Texten, “um publicado no programa da primeira encenação de Orfeu da Conceição no Teatro Municipal do Rio, o outro inédito, escrito provavelmente para o Festival de Cannes, em forma de testemunho". (Moraes, Radar, 47)

${ }^{9}$ Armbruster weist auf diese Differenz zwischen Text und Paratext hin. Das Stück spreche der afrobrasilianischen Kultur den Status von Hochkultur ab, während der Paratext den Afrobrasilianer auf eine Stufe mit der griechischen Hochkultur zu stellen versuche (Armbruster, "'Verhandlungen' über das afrikanische Brasilien auf der Bühne: Das Theaterstück Orfeu da Conceição (1954) von Vinicius de Moraes”, 34). 
a beber, insinuando, criando a festa" (Moraes, Orfeu, 83). Dieser Akt erweist sich als Loblied auf brasilianische Musik, denn hier werden einige für den Samba charakteristische Musikinstrumente feierlich vorgestellt, die in einem zweiten Schritt zu einem Samba zusammenfinden. Unterbrochen wird dieses Spektakel durch Orfeu, dessen Gitarrenklänge und der Ruf nach Eurídice die Feiernden innehalten lassen. Seine Musik lässt sogar den Höllenhund Cérbero zurückweichen.

Im dritten Akt kehrt Orfeu schließlich ohne Eurídice auf seinen morro zurück. Der Chor verkündet, dass er aus Liebe verrückt geworden sei, wie auch seine Mutter Clio, die sein Schicksal nicht verkraftet. In einer dramatischen Schlussszene wird Orfeu zum Opfer einer Horde wild gewordener Frauen, die ihn ermorden. Nach dieser "carnificina" zerschmettert Mira Orfeus Gitarre und ein Chor, der die Unsterblichkeit des Musikers betont, schließt die Szene ab: "Tudo morre que nasce e que viveu/ Só não morre no mundo a voz de Orfeu" (Moraes, Orfeu, 109).

Durch sein Stück, das er als "homenagem [...] ao negro brasileiro" (Moraes, Radar, 49) bezeichnete, aber auch aufgrund seiner legendären Inszenierung mit schwarzen Schauspieler*innen erhielt Vinicius, der sich übrigens selbst als "o branco mais preto do Brasil" (Costa e Silva 217) bezeichnete, den Ruf "eines der afro-brasilianischen Welt nicht nur zugeneigten, sondern sogar zugehörigen Dichters” (Armbruster, “'Verhandlungen' über das afrikanische Brasilien auf der Bühne: Das Theaterstück Orfeu da Conceição (1954) von Vinicius de Moraes”, 35). Seine Forderung "Todas as personagens da tragédia devem ser normalmente representadas por atores da raça negra, não importando isto em que não possa ser, eventualmente, encenada com atores brancos" (Moraes, Orfeu, 54) erscheint aus heutiger Perspektive eher problematisch, zumal es eine Absage an mestizische Darsteller*innen ist, ${ }^{10}$ aus historischer Perspektive allerdings "hat

10 Armbruster versucht Vinicius' Streben nach homogenen Darsteller*innen nachzuvollziehen: "In der Tragödie sollen sich die Zuschauer alleine auf die 
diese Regieanweisung sicherlich eine gutgemeinte, affirmative Ausrichtung, da afrobrasilianische Figuren im Theater kaum vorhanden waren” (Armbruster, “'Verhandlungen' über das afrikanische Brasilien auf der Bühne: Das Theaterstück Orfeu da Conceição (1954) von Vinicius de Moraes", 26).

\section{Auf dem morro: Favela und Slang}

Orfeu da Conceição zählt als erstes brasilianisches Theaterstück, das eine Favela thematisiert (Oliveira, 143). Spätestens seit Paulo Lins' Roman Cidade de Deus (1997) und der gleichnamigen international erfolgreichen Verfilmung von 2002 hat auch ein breiteres deutsches Publikum Einblicke in Aufbau, Strukturen und Alltag einer brasilianischen Favela erhalten, allerdings sind spezifische Kenntnisse und die Vertrautheit mit bestimmten Begriffen eher nicht zu erwarten. Eine Herausforderung für die Übersetzung ins Deutsche stellen damit erstens Begriffe aus einem semantischen Feld dar, das auf einen spezifisch brasilianischen soziokulturellen Kontext verweist, nämlich die favela carioca, und zweitens ist fraglich, wie der "Favela-Slang", auf den Vinicius gleich zu Beginn seines Stücks verweist, in die deutsche Sprache übersetzt werden kann.

Schauplatz des Stücks ist "um morro carioca" (Moraes, Orfeu, 55 ${ }^{11}$, der als Szene zu Beginn des Stücks im Kontrast zur

Inhalte konzentrieren, ohne durch unterschiedliche ,gemischte" Hautfarben auf eine andere Interpretationsebene $\mathrm{zu}$ gelangen. Allerdings spricht aus diesen Regieanweisungen auch eine Form von Aussparung möglicher ethnischer Konflikte oder Benachteiligungen. Denn, wenn es nicht möglich ist, dass weiße und schwarze Schauspieler gleichzeitig auf der Bühne spielen, ohne dass die Gedanken sich um die ethnische Komponente drehen, bereitet der ethnische Kontext wohl doch Probleme" (Armbruster, “'Verhandlungen' über das afrikanische Brasilien auf der Bühne: Das Theaterstück Orfeu da Conceição (1954) von Vinicius de Moraes”, 25).

${ }^{11}$ Dass es sich um einen Favelahügel in Rio de Janeiro handeln soll, betont Vinicius auch in Radar da Batucada: "A ação situa-se no tempo presente, num morro que poderia ser não importa qual da cidade" (Moraes, Radar, 49). 
Stadt beschrieben wird und damit die Antithese von favela und asfalto bekräftigt: "O morro, a cavaleiro da cidade, cujas luzes brilham ao longe. Platô de terra com casario ao fundo, junto ao barranco, defendido, à esquerda, por pequena amurada de pedra, em semicírculo, da qual desce um lance de degraus. Noite de lua, estática, perfeita. No barranco de Orfeu, ao centro, bruxuleiam lamparinas" (Moraes, Orfeu, 55). Morro kann in diesem Kontext als Synonym für Favela und damit als Antithese zur nicht nur in geografischer Ferne liegenden Stadt betrachtet werden. Erst im zweiten Akt verlässt Orfeu seine Favela, um in die Stadt hinabzusteigen, wo der höllische Karneval wütet. Die Stadt wird damit zum "inferno da favela" (Calil 12). Der morro entpuppt sich als zentrales Motiv des Stücks: Bereits in der Aufzählung der Personen heißt es "Mira de tal, mulher do morro" oder "gente do morro", um ihre Bindung an den Favelahügel zu betonen. In einer Szene mit der Schwarzen Dame betont Orfeu seine Zugehörigkeit und Wirkungsmacht als Musiker auf dem morro:

\footnotetext{
Vá embora

Senhora Dama! eu lhe digo: vá embora!

No morro manda Orfeu! Orfeu é a vida

No morro ninguém morre antes da hora!

Agora o morro é vida, o morro é Orfeu

É a música de Orfeu! Nada no morro

Existe sem Orfeu e a sua viola!

Cada homem no morro e sua mulher

Vivem só porque Orfeu os faz viver

Com sua música! Eu sou a harmonia

E a paz, e o castigo! Eu sou Orfeu

O músico!
}

(Moraes, Orfeu, 76)

Setzt Vinicius morro hier repetitiv ein, stellt sich die Frage nach einer angemessenen deutschen Übersetzung, impliziert der Begriff doch eine spezifisch brasilianische soziale Realität: "Hügel” allein 
reicht bestimmt nicht aus, um die im Portugiesischen implizite Verknüpfung von Favela und Topografie, nämlich die hügelige Landschaft Rio de Janeiros, aber auch den Kontrast zur Stadt deutlich zu machen. "Favelahügel" klingt vergleichsweise etwas umständlich und wird Vinicius' Reimen bestimmt nicht gerecht, doch kommt damit die dem portugiesischen Begriff inhärente Beziehung zwischen Favela und Topografie zum Ausdruck. Morro wird also in der deutschen Übersetzung zu Favelahügel:

Vá embora

$\mathrm{Hau} \mathrm{ab}$

Senhora Dama! eu lhe digo: vá Schwarze Dame! Ich sage Dir: embora! $\mathrm{Hau} \mathrm{ab}$ !

No morro manda Orfeu! Orfeu é Auf dem Favelahügel befiehlt a vida Orfeu! Orfeu ist das Leben

No morro ninguém morre antes da Auf dem Favelahügel stirbt hora! niemand vorzeitig!

Agora o morro é vida, o morro é Nun ist der Favelahügel Leben, Orfeu der Favelahügel ist Orfeu

É a música de Orfeu! Nada no Ist die Musik von Orfeu! Nichts morro auf dem Favelahügel

Existe sem Orfeu e a sua viola! Existiert ohne Orfeu und seine Gitarre!

Cada homem no morro e sua Jeder Mann auf dem Favelahügel mulher und seine Frau

Vivem só porque Orfeu os faz Leben nur, weil Orfeu sie lebendig viver macht

Com sua música! Eu sou a Durch seine Musik! Ich bin die harmonia Harmonie

E a paz, e o castigo! Eu sou Orfeu Und der Frieden und die Strafe! Ich bin Orfeu

O músico!

Der Musiker!

Bereits der Titel des Stücks - Orfeu da Conceição. Tragédia carioca em três atos - spielt vermutlich auf den Morro da 
Conceição an. ${ }^{12}$ Hier wäre es allerdings sinnvoll, den Titel für ein deutsches Publikum, das nicht mit Rio de Janeiros Topografie vertraut ist, plakativer zu gestalten, z.B. Orpheus in der Favela. Allerdings sollte der Originaltitel beispielsweise in einer Fußnote erklärt werden. Der Untertitel "Tragédia carioca" lässt sich wohl am besten mit "Eine Tragödie aus Rio de Janeiro" übersetzen.

Orfeu da Conceição wurde zwar in den 1940/50er Jahren geschrieben, sieht als "Tempo" in der Szenenbeschreibung allerdings "O presente" (Moraes, Orfeu, 55) vor, was Vinicius' Wunsch nach einer regelmäßigen Aktualisierung bereits andeutet. Das Stück kombiniert Hoch- und Umgangssprache: Einerseits versammelt es poetische Musikstücke des Lyrikers Vinicius, die allerdings auch gerne umgangssprachlich verfahren, andererseits versucht das Stück einen Favela-Slang der 1950er Jahre abzubilden. Wie Vinicius in der Szenenanweisung betont, spielt der Slang eine bedeutende Rolle und sollte bei einer Inszenierung angepasst werden: "Tratando-se de uma peça onde a gíria popular representa um papel muito importante, e como a linguagem do povo é extremamente mutável, em caso de representação deve ela ser adaptada às suas novas condições" (Moraes, Orfeu, 54). Vor allem Mira und die anderen Prostituierten vom morro lässt Vinicius Umgangssprache verwenden:

A PRIMEIRA MULHER

E Mira, ocê já viu? Tá doida, Mira...

\section{DIE ERSTE FRAU}

Und Mira, haste die schon gesehen?

Total verrückt, diese Mira...

Neben zahlreichen umgangssprachlichen Satzkonstruktionen finden sich auch einige umgangssprachliche Begriffe. Ein Beispiel für diesen Favela-Slang ist der Begriff neguinho bzw. neguinha, der von Eurídice für Orfeu und von Orfeu für Eurídice

${ }^{12}$ Die Idee zu dem Titel kam von João Cabral (Castello 126). 
verwendet wird. Das Diminutiv von nego bzw. nega wird bei der Übertragung ins Deutsche vor ein doppeltes Problem gestellt: Die wörtliche Übersetzung, Negerlein, gilt im Deutschen nicht nur als diskriminierend und veraltet, auch die dem portugiesischen Begriff inhärente emotionale Komponente, nämlich die Funktion als Kosewort bzw. liebevolle Anrede ließe sich zwar durch das Diminutiv erahnen, wird aber kaum verständlich. In der italienischen Übersetzung von 1961 hat Pasquale Ariel Jannini neguinho mit mio caro oder amore mio übersetzt (Jannini 53,55,77). Mit dieser Entscheidung für einen gängigen italienischen Kosenamen hat er das Problem der schwarzen Komponente des Begriffs umgangen. Im Hinblick auf die afrobrasilianische Thematik des Theaterstücks ist diese Lösung allerdings ziemlich unbefriedigend. Wie lässt sich also neguinho bzw. neguinha ins Deutsche übertragen, ohne den für das Theaterstück - relevanten Verweis auf eine schwarze Person zu verlieren und - Vinicius' Anliegen nach einer Aktualisierung folgend - ohne aus heutiger Perspektive veraltet zu klingen? Tatsächlich scheint hier eine Entlehnung aus dem brasilianischen Portugiesischen am unkompliziertesten. Auch unterstreicht eine Beibehaltung von neguinho bzw. neguinha in der deutschen Übersetzung den spezifisch brasilianischen Charakter des Stücks.

\section{Samba}

Der Orpheus-Mythos ist ein Loblied auf die Musik: Erst durch seinen Gesang und das Spiel seiner Lyra gelingt es Orpheus Hades, Persephone und Kerberos zu besänftigen und Eurydike zurückzugewinnen. Vinicius de Moraes' Rezeption nimmt dieses Motiv besonders ernst und überträgt es auf einen brasilianischen Kontext: Orfeu da Conceição ist ein Musiktheaterstück mit "Samba"-Einlagen, so werden diese in den Szenenanweisungen jedenfalls bezeichnet. Ursprünglich im Rahmen einer "homenagem [...] ao negro brasileiro" (Moraes, Radar, 49) als Hymne auf afrobrasilianische Musik gedacht, wurden diese Musikstücke 
später zur Anfangsphase des Bossa Novas erklärt. ${ }^{13}$ Der Hinweis, dass afrobrasilianische Rhythmen, batuques, die als Ursprung des Sambas zu betrachten sind, seine Kompositionen beeinflusst haben, war Vinicius besonders wichtig: Bereits in dem dem Stück vorangestellten Text Radar da Batucada erklärt er eine TrommelPercussion vom nahegelegenen Favelahügel zum Ausgangspunkt für seine zündende Idee zu einem "schwarzen Orpheus". Wie Diniz im Almanaque do samba betont, steht der Samba nicht nur symbolisch für die Integration schwarzer Kultur, sondern ist in der Entstehungszeit von Orfeu da Conceição zum zentralen Charakteristikum brasilianischer Kultur avanciert:

\begin{abstract}
O samba foi o recheio, por vezes inspiração, de quase todos os movimentos musicais desta terra carnavalesca. Apesar de ser um gênero resultante das estructuras musicais europeias e africanas, foi com os símbolos da cultura negra que o samba se alastrou pelo território nacional. No passado, os viajantes denominavam batuque qualquer manifestação que reunisse dança canto e uso de instrumentos dos negros. Esse era então um termo genérico para designar festejos. [...] Na década de 1940, o samba passa a ser sinônimo de brasileiro e ganha fama internacional, de forma que hoje o mundo inteiro vê o Brasil como berço do carnaval e do samba. (Diniz 13-14)
\end{abstract}

Orfeu da Conceição thematisiert sowohl Samba als auch Karneval und stellt damit die Frage nach afrobrasilianischer Kultur. Wie lassen sich die Samba-Stücke allerdings ins Deutsche übertragen? Berühmte Songs wie Um nome de mulher, Se todos fossem iguais a você, Mulher, sempre mulher, Eu e o meu amor, Lamento do

${ }^{13}$ Vinicius gilt als "padre putativo (con Antônio Carlos Jobim y João Gilberto) de la bossa nova" (Campos-Muñoz 33). Als offizielles Debütjahr des Bossa Nova gilt 1959 mit der Veröffentlichung der LP Chega de Saudade, von João Gilberto. Der Song wurde von Jobim komponiert, die Lyrics stammen von Vinicius. 
morro haben mittlerweile internationale Popularität erreicht. Aus diesem Grund, dem spezifisch brasilianischen Charakter und nicht zuletzt wegen der formalen Gestaltung der Lyrics - Vinicius schien Metrik häufig wichtiger zu sein als Inhalt (Castello 186) - macht eine Übersetzung dieser Songs nur als Zusatz Sinn. Mindestens für die Songs, wenn nicht auch für das gesamte Stück - im Sinne einer bilingualen Ausgabe - wäre die Kombination von Original und Übersetzung zweifellos eine Bereicherung. Eine mögliche Inszenierung könnte mit Original und Übersetzung in Form von Unter- oder Übertiteln arbeiten. Dass es allerdings schier unmöglich scheint, Inhalt und Form gleichermaßen gerecht zu werden ${ }^{14}$ und dass die Übersetzerin im Hinblick auf die Form kreativ werden muss, soll anhand einer Übersetzung des Songs Mulher, sempre mulher, den Orfeu im zweiten Akt nach einem Streit mit Mira singt, exemplarisch gezeigt werden:

Mulher, ai, ai, mulher

Sempre mulher dê no que der

Você me abraça, me beija, me xinga

Me bota mandinga

Depois faz a briga

Só pra ver quebrar!

Mulher, seja leal

Você bota muita banca
Frau, au, au, Frau

Für immer Frau, komme, was komme

Du umarmst mich, küsst mich, beschimpfst mich

Du verzauberst mich

Dann nimmst Du den Kampf auf

Nur um zu sehen, wie alles zerbricht!

Frau, sei treu

Du hältst dich für den Nabel der Welt

E infelizmente eu não sou jornal. Und leider ist kein Platz für mich.

Mulher, martírio meu

O nosso amor
Frau, meine Qual

Unsere Liebe

\footnotetext{
${ }^{14}$ Einer der wenigen Übersetzer von Vinicius' Gedichten und Songs, Kay-Michael Schreiner, erklärt, dass er sich bemüht habe, dem Rhythmus des Originals zu folgen, auf Reim habe er dagegen weitgehend verzichtet (Schreiner 8).
} 
Deu no que deu

E sendo assim não insista, desista

Vá fazendo a pista

Chore um bocadinho

E se esqueça de mim.

(Moraes, Orfeu, 73)
Es kam wie es kam

Und wenn es so ist, bestehe nicht darauf, sondern gib auf

$\mathrm{Hau} \mathrm{ab}$

Weine ein Weilchen

Und vergiss mich.

Der Slang zeigt sich auch hier erneut als Herausforderung. Bei der Übersetzung des Ausdrucks "dê no que der" wurde auf die im Deutschen korrekte Redewendung ("komme, was wolle") zu Gunsten einer von Vinicius intendierten Wiederholung des Verbs ("komme, was komme") verzichtet. Dies ist auch im Hinblick auf die erneute Wiederholung von "dar" ein paar Zeilen später ("deu no que deu") sinnvoll. Reime werden verlagert, wie zum Beispiel im Fall von "não insista, desista", das zu "bestehe nicht darauf, sondern gib auf" wird. Der umgangssprachliche Ausdruck "botar banca" wird mit "sich für den Nabel der Welt halten" übersetzt, wodurch die Alliteration allerdings verloren geht. Andere Ausdrücke wie "fazer a pista" oder "bocadinho" finden im Deutschen mit "abhauen" bzw. "Weilchen" ein treffendes Äquivalent. Wieder wird deutlich, dass sich Vinicius' zahlreiche Reimspiele kaum ins Deutsche übertragen lassen.

Nicht nur die Übersetzung der Musikstücke erweist sich als Herausforderung, auch Begriffe aus dem semantischen Feld afrobrasilianischer Musik lassen sich nicht übersetzen und benötigen eine Erklärung. Im zweiten Akt des Stücks, in der karnevalesken Unterwelt, werden Zusammensetzung und Aufbau des Sambas durch die Karnevalsgesellschaft thematisiert, womit eine intendierte kulturvermittelnde Funktion des Stücks besonders hervortritt. Bevor sich Orfeu dem "clube dos Maiorais" mit seiner Gitarre nähert, stellt Plutão verschiedene für den Samba charakteristische Percussion-Instrumente und ihre Funktion für den Samba der Karnevalsgesellschaft vor: 
PLUTÃO

Quem é que marca o tempo, meus filhos?

TODOS

É o bumbo!

Ouve-se o som monstruosamente ampliado de um bumbo.

PLUTÃO

Quem é que marca o ritmo?

TODOS

É o tamborim!

PLUTÃO

O mesmo, com um tamborim.

Quem é que marca a cadência?

TODOS

É o pandeiro!

O mesmo, com um pandeiro.

(Moraes, Orfeu, 84-85)

So geht es eine Weile weiter, hinzu kommen cuíca, brincadeira und agogô bis Plutão schließlich fragt: "Então como é como é como é? Sai ou não sai esse samba?" (Moraes, Orfeu, 85) und Orfeus Gitarre den Höllenlärm irritiert. Diese Eigennamen der einen Samba konstituierenden Instrumente werden in der deutschen Übersetzung beibehalten. Die Instrumente lassen sich mit Hilfe von Fußnoten kurz erklären, dazu zählen auch weitere für das Stück relevante Begriffe wie choro, ciranda, batuque bzw. batucada. Für letzteren bietet sich beispielsweise eine einfache Erklärung aus dem Novo Aurelio Século XXI an: "Reunião popular, geralmente nas ruas, onde se toca o samba em instrumentos de percussão, com acompanhamento vocal ou sem ele."

Zum semantischen Feld des Sambas zählt der Karneval. Ebenso wie der Samba steht auch der Karneval für die Verbindung von schwarzer Kultur und nationaler Symbolik:

If one considers that there is a strong sense of belonging amongst old and mostly black sambistas towards carnival 
festivities, one has also to consider the possibility of politics associated with the issue of recognition. The carnival feast has given voice to many black people whose artistic productions were widely recognized as symbols of the nation. (Santos 111)

Für den brasilianischen Karneval relevante Begriffe müssen einem deutschen Publikum ebenso vermittelt werden. Im zweiten Akt heißt es beispielsweise:

PLUTÃO (bradando)

Alegria! É o reinado da alegria! Amanhã é Cinzas! Hoje é o último dia! E viva Momo! E viva a folia!

(Moraes, Orfeu, 87)

\section{PLUTO (schreiend)}

Freude! Ein Königreich der Freude! Morgen ist Aschermittwoch! Heute ist der letzte Tag! Es lebe Momo! Es lebe die Gaudi!

Symbolfigur des Karnevals ist "Rei Momo", der den Karneval eröffnet. Die ursprünglich aus der griechischen Mythologie stammende Figur "Momos" ist Namensgeber für Begriff und Figur aus dem Theaterbereich, nämlich "pequena farsa popular" bzw. "o ator que representava nessas farsas; bufo" bzw. in einem brasilianischen karnevalesken Kontext "Figura inspirada em momo, e que personifica o carnaval" (Novo Aurelio Século XXI).

Das Stück spielt auf einige weitere afrobrasilianische Traditionen an. In einer Hintergrundszene lässt Vinicius zwei malandros capoeira praktizieren und die Dama Negra tanzt zu Orfeus Musik "passos de macumba" (Moraes, Orfeu, 77). Auch diese Begriffe lassen sich nicht übersetzen und werden in Form von Fußnoten in der deutschen Übersetzung erklärt. 


\section{Orpheus in der Favela}

Vinicius Mariano de Carvalho inszenierte 2016 während des Festival of Arts and Humanities am King's College in London eine Performance mit dem Titel Playing with Orpheus, wofür er Orfeu da Conceição als Vorlage verwendete. Vor allem Songs, einige von Orfeus' Monologen und ein paar Teile des Chors wurden in diesem Rahmen auch übersetzt. Zur Inszenierung erklärt Carvalho: "My concept was to avoid the framework of the antithetical pair of morro-cidade. There was no stage, properly speaking. Audience, musicians and the singer were circularly distributed. The middle of the circle was the arena for the capoeira and the maracatu groups" (Carvalho 595). Carvalhos Inszenierung zeigt, dass Vinicius' Stück für ein zeitgenössisches Publikum interessant ist und neue Interpretationen gewagt werden. Die Auflösung des antithetischen Paars morro-cidade im Rahmen einer Inszenierung kann als kreative Zukunftsvision betrachtet werden, wobei diese Auflösung im Rahmen einer Übersetzung sicherlich zu weit ginge.

Der Beitrag hat versucht, ein paar Herausforderungen für die deutsche Übersetzung zu skizzieren: Vor allem für die Vermittlung afrobrasilianischer Kultur müssen Strategien entwickelt werden. Im Fall von Begriffen, die auf einen spezifisch brasilianischen soziokulturellen Kontext verweisen, bietet es sich an, einige Begriffe im Original zu belassen, so zum Beispiel das Diminutiv neguinho/neguinha, spezifische Musikinstrumente des Sambas, aber auch capoeira oder macumba. Eine weitere Möglichkeit stellt die Anpassung eines Begriffs im Deutschen (Favelahügel) dar, um die soziokulturelle Bedeutung von der Ausgangssprache in die Zielsprache zu transportieren. Diese Strategien tragen dazu bei, den spezifisch brasilianischen bzw. afrobrasilianischen Charakter des Stücks auch in der Übersetzung zu betonen.

Eine Übersetzung kann die raffinierte formale Gestaltung der zahlreichen Songs des Stücks, wie auch andere Teile und ihre Verse, kaum berücksichtigen. Aus diesem Grund, aber auch aufgrund der internationalen Popularität der Musikstücke bietet 
sich eine Ergänzung der Übersetzung durch das Original an. Überhaupt wäre zu überlegen, ob eine bilinguale Ausgabe für das gesamte Stück von Vorteil wäre.

Dem Paratext, also Begriffe erläuternde Fußnoten, eine kulturhistorische, interpretatorische und biografische Informationen zusammenstellende Einleitung, sowie eine Übersetzung von Radar da Batucada, kommt in dieser Übersetzung eine bedeutende Rolle zu: Er übernimmt eine zentrale kulturvermittelnde Funktion für ein deutsches Lesepublikum.

Nicht zuletzt ist mit der Übersetzung ins Deutsche auch eine Hoffnung verbunden: Die Übersetzung wäre der erste Schritt zu weiteren Inszenierungen. Schön wäre es, wenn Orfeu da Conceição es als Orpheus in der Favela bald auf Bühnen im deutschsprachigen Raum schafft.

\section{Bibliografie}

Armbruster, Claudius. "Neue Heimat, altes Elend - Das Eigene und das Fremde in brasilianischen und europäischen Diskursen über die Favela". Heimat in der Fremde. Pátria em terra alheia, Henry Thorau (Ed.), Berlin: edition tranvía Verlag Walter Frey, 2007, pp. 357-389.

Armbruster, Claudius. “'Verhandlungen’ über das afrikanische Brasilien auf der Bühne: Das Theaterstück Orfeu da Conceição (1954) von Vinicius de Moraes”. Grenzüberschreitungen in der Lusophonie. Intermediales - Intertextuelles Interkulturelles, Claudius Armbruster (Ed.), Hamburg: Verlag Dr. Kovač, 2012, pp. 17-39.

Calil, Carlos Augusto. "Fantasia do poeta". Vinicius de Moraes: Teatro em versos. organizado por Carlos Augusto Calil. São Paulo: Companhia das Letras, 1995, pp. 9-16. 
Campos-Muñoz, Germán. "Contrapuntos ótficos: Mitografía brasileña y el mito de Orfeo". Latin American Research Review 47. Special Issue, (2012): 31-48.

Carvalho, Vinicius Mariano de. "The Metamorphoses of Vinicius de Moraes' Orfeu da Conceição”. Brasiliana. Journal for Brazilian Studies 9.1, (2020): 580596.

Castello, José. O poeta da paixão. Uma biografia. São Paulo: Companhia das Letras, 1994.

Costa e Silva, Paulo da. "Da poesis à canção: Notas sobre o Vinicius de Moraes letrista”. Vinicius de Moraes: Livro de Letras, Eucanaã Ferraz (Ed.), São Paulo: Companhia das Letras, 2015, pp. 209-219.

Diegues, Carlos. "Conceição a 40 graus. Carnavalização, a lógica do espetáculo e a palavra-chave do Século 20". Cinemais 17. (1999): 7-47.

Diniz, André. Almanaque do samba. Rio de Janeiro: Jorge Zahar, 2006.

Ferreira, Aurélio Buarque de Holanda. Aurelio Século XXI. O dicionário da língua portuguesa. Rio de Janeiro: Nova Fronteira, 1999.

Moraes, Vinicius de. Orfeo Negro. Tradução de Pasquale Aniel Jannini. Milano: Nuova Accademia Editrice, 1961.

Moraes, Vinicius de. "Ein Gespräch mit Vinicius. Das Leben ist wichtiger als die Kunst". Vinicius de Moraes: Saravá. Gedichte und Lieder, selecionado e traduzido por Kay-Michael Schreiner, Frankfurt: Vervuert, 1982, pp. 111-118.

Moraes, Vinicius de. "Orfeu da Conceição". Vinicius de Moraes: Teatro em versos, organizado por Carlos Augusto Calil, São Paulo: Companhia das Letras, 1995, pp. 51-112.

Moraes Vinicius de: "Radar da Batucada". Vinicius de Moraes: Teatro em versos, organizado por Carlos Augusto Calil, São Paulo: Companhia das Letras, 1995, pp. 47-49. 
Oliveira, Marina de. "A favela em Orfeu da Conceição: poetização e eurocentrismo”. Navegações 5.2, (2012): 143-148.

Porra, Véronique: "Sur quelques Orphée noirs. Reproduction, adaptation et hybridation du mythe d'Orphée en contexte post (-) colonial". Revue de Littérature Comparée 4, (2012): 441-455.

Schreiner, Kay-Michael. "Vorwort". Vinicius de Moraes: Saravá. Gedichte und Lieder, selecionado e traduzido por Kay-Michael Schreiner, Frankfurt: Vervuert, 1982, pp. 7-8.

Santos, Myrian Sepúlveda dos. "Black Orpheus and the Merging of two Brazilian Nations". European Review of Latin American and Caribbean Studies 71. October, (2001): 107-116.

Recebido em: 07/04/2021

Aceito em: 28/07/2021

Publicado em setembro de 2021

Martina Kopf. E-mail: kopfm@uni-mainz.de. ORCID: https://orcid.org/00000001-5793-1330. 